\title{
The Diagnosis of Early Extra-Uterine Pregnancy.*
}

\author{
By W. Axstruther Mridigan, M.B. (Aberd.), F.R.C.S. (Edin.), \\ Late Registrar, Hospital for Women, Soho Square, London.
}

THE diagnosis of cases of early extra-uterine gestation presents many points of vast importance and of extreme interest. Our knowledge of this condition, within recent years, has very much increased, and now there is a considerable number of cases on record of the correct diagnosis of "Unruptured tubal gestation."

During the last three and a half years I have had the opportunity of closely observing some fifty cases of extra-uterine gestation, and others which have been considered such until operation or subsequent history proved that they were not. It is largely on a study of these that the following remarks are based. The term extra-uterine, and not ectopic, is used, as interstitial pregnancies are not touched on at all. The expression "early" is meant to cover such cases as are presented before there is a possibility of hearing any foetal heart sounds.

The most common time in a woman's life for her to be the subject of an extra-uterine gestation corresponds to the most active period of her sexual life, i.e., between the ages of twenty-five and thirty-five. The actual time after marriage for such a pregnancy to occur varies greatly. It must be remembered that it may occur at any time after marriage, although it is not common until after several (eight to ten) years of married life. Any question as to previous sterility has to my mind no significance in the weighing of the "pros and cons" of an extra-uterine gestation. In my series the average number of previous pregnancies was four and the average time since last pregnancy was four and a half years, neither of which facts would permit the women to be classed as sterile. Extra-uterine gestation is more common in multiparous women, and is more likely to follow a full-time pregnancy than a miscarriage or abortion. It may, however, occur the first time a woman becomes pregnant, as it did in eight of my cases.

In discussing the diagnosis the subject will be divided thus:-

* Amplified from a paper read before the Hunterian Society of London, Feb. 28, 1906. 
I. What the patient tells:

(1) In her own words.

(2) In answer to more minute enquiry.

II. What may be expected to be found on examination.

III. The most likely things that may be confused with an extra-uterine pregnancy.

\section{What the Patient Telis.}

(1) In her own words.

Pain is almost certain to be the symptom for which advice is sought. The actual situation and character of this pain vary much. It is most usually situated in the lower part of the abdomen at one or other side, or may be located in the back. Sometimes it is referred to more distant parts, but this is unusual. The character is described as of varying degrees of intensity, starting possibly with a sudden sharp attack, which lasted a short time and then passed off, leaving more of a dull ache in the side. Such sharp attacks may recur at periodic intervals. Rest gives little, if any, relief to the pain. One very important point with regard to the pain is, that it may possibly be referred entirely to the act of micturition. This is due to pressure effects and seems to occur in those cases where the ovum becomes lodged at an early stage in front of or behind the uterus.

In addition to the history of pain the patient states that she went over her usual time for menstruation, perhaps a few days or a few weeks, or else she will report that she has been perfectly regular; but that with the pain she "came on unwell" again, and that she has been "unwell on and off since." In the latter case she will have no idea that she is pregnant.

(2) In answer to more minute enquiries.

(a) With regard to pain. It is very necessary that particular notice should be taken of this symptom, as a correct interpretation of the pain very often leads to a right diagnosis. The onset is often attributed to a knock, a fall, or some such injury, and even when the attack commences at night, a heavy day's work is usually assigned as the cause. For some years previously the patient may have complained of pain, and will now merely say that it has got worse recently. Patients do not separate this recent pain from the chronic pain they have had for years. Nearly always, however, on enquiry, the two kinds of pain can be separated, and the more recent 


\section{Milligan: Extra-Uterine Pregnancy}

one is found to be very much more distinct in character and severity than the chronic pain.

The acute exacerbations of colicky pain are sometimes not easy to get a distinct history of. When such can be elicited they form very conclusive evidence in support of the presence of an extra-ntorine gestation.

(b) With regard to the bleeding pervaginam, most minute enquiry has to be made, as this is sometimes the one symptom on which a diagnosis is based. It is necessary in the first place to be extremely careful not to allow the fact, that the history of having "never missed a period" is given, to discountenance the idea that the condition is one of extra-uterine gestation. In nearly 50 per cent. of my cases the history given was that no period had been missed, but minute enquiry elicited in the majority of cases the fact that the last period had not been quite like former ones.

In this connection it is interesting to note what J. W. Taylor says with regard to this amenorrhoea. In the Lancet, 1892 , he writes, "Where one finds a history of amenorrhcea, followed by irregular loss, together with signs of a tubal tumour, in a woman of child-bearing age who has been previously healthy, there is every reason to suspect that extra-uterine pregnancy has begun." In the Medical Press and Circular, July 18th, 1894, he adds to the above, "Where the other signs are present, but the history of amenorrhœa is wanting, the diagnostic probability lies in favour of so-called 'tubal abortion." "

That some irregularity in the way of hamorrhagic discharge is an important, some say invariable, symptom, there is no doubt. 'The chief point to elicit is, whether or not, if no period has been missed, the bleeding that has come on with the pain is like the bleeding of an ordinary period or whether it is different, and if so, in what does it differ.

Should a period of amenorrhcea have preceded the hrmorrhagie discharge, then the same points have to be determined, as in those cases where there has been no amenorrhœa.

These points are-

(1) The colour.

(2) The persistence.

(3) The presence of membrane or pieces of membrane.

(1) Colour. As pointed out by Cullingworth, 3 the discharge is dark in colour, almost blackish, and not a bright red discharge as from direct arterial bleeding. In this condition the blood comes from a large surface, more as a general ooze, than as bleeding 
from any one special point. Some of the blood may be retained some time before it is passed and is therefore not fresh blood. This dark colour of the discharge is a very characteristic point in the hæmorrhage accompanying an extra-uterine gestation.

(2) Persistence. A hæmorrhagic discharge that keeps on day after day and not ceasing as the hæmorrhagic discharge from an ordinary period does after a certain number of days, is very characteristic. The actual amount lost may vary considerably, sometimes the discharge stopping for a time, only to recur again. Then there may be "floodings" every now and again during the course of this persistent discharge.

It is to be noted that any acute attack of pain frequently increases the amount of discharge for the time being.

(3) The passage of membrane or pieces of membrane. In this there is almost a pathognomonic sign of extra-uterine gestation, if examination of the membrane reveals the presence of decidual cells, and no chorionic villi or fotal remnants. Decidual cells are found in response to a pregnancy, and hence, if present, the pregnancy must be either intra-uterine or extra-uterine (including tubo-uterine). If the pregnancy is intra-uterine, then chorionic villi or some foetal remains should be found.

In this connection it must be remembered that cases have been reported in which decidual casts have been passed and yet extrauterine pregnancy has been proved not to exist. Such cases have been reported by Griffith, ${ }^{7}$ Dakin, ${ }^{4}$ and Eden. ${ }^{5}$ Thus there are three cases in which a cast was passed having all the characters of an extra-uterine cast, and yet no extra-uterine pregnancy existed. The aeceptance of these cases involves the giving up of the idea that decidual tissue is formed only in response to a fertilised ovum. Until further proof is forthcoming it must be held that the passage of a cast $0 I^{*}$ pieces of membrane, having the characteristics of an extra-uterine gestation cast, is very strong evidence in favour of the fact that the patient is the subject of an extra-uterine gestation. Cullingworth ${ }^{3}$ lays particular stress on the importance of this as helping to clinch the diagnosis in a doubtful case.

\section{What MAY be EXPECTED to BE FOUND ON EXAMINATION.}

In certain urgent cases where hæmorrhage is proceeding and the patient is evidently dying from such internal hæmorrhage, the diagnosis is rendered certain by the operation which is undertaken as the only possible means of saving life. In these cases the hæmorrhage is intra-peritoneal, either from a rupture in the tube or from a 
hæmorrhage through the abdominal ostium of the tube. Taylor ${ }^{10}$ says, in recording a case of early rupture, where on examination he could find no tumour or physical sign pointing to a misplaced pregnancy: "I did not know at that date that in some cases of very early rupture this may be entirely wanting" (p. 94). In cases where symptoms are not so urgent there is the advantage of getting a history and of making a thorough examination. It may be that in many of such cases an anæsthetic is called for, in order to be thoroughly satisfied as to what is in the pelvis. In passing it is important to note that extreme gentleness in examination is essential, as cases are recorded where a tube has ruptured during an examination, presumably on account of the pressure exerted. In this examination there is no question as to the great help afforded by the abdomino-recto-vaginal examination. In some cases very little may be felt owing to the blood not having had time to organize, but usually very distinct signs are forthcoming, and no sign must be left unlooked for that might be expected to be present in the case of an extra-uterine gestation. The chief point is to make sure that there is something in the pelvis separate from the uterus. Let the uterus be clearly defined and then a swelling to one or other side differentiated or not as the case may be.

Softening of the cervix certainly takes place during the progress of an extra-uterine gestation, but in the early months it is difficult to place any real reliance on this as a point in the diagnosis. A more frequent and certain sign is distinct pulsation in one or other fornix, corresponding to the side on which the gestation lies. Then, as to the uterus, that it enlarges gradually during the evolution of an extra-uterine gestation is certain, but never to the extent corresponding to the enlargement where the pregnancy is intra-uterine. In the early months, therefore, it is a sign to be carefully looked for, but in assigning to it its value, it must always be remembered that the enlargement may be from other causes, e.g., chronic metritis.

The passage of a sound may in certain cases be necessary and justifiable, and when so, will afford definite proof of the condition of the uterus, as to whether there is any enlargement, and as to whether it is empty or not.

In the case of an unruptured tubal pregnancy, examination will, most probably, in conjunction with the symptoms, enable a correct diagnosis to be made. To one or other side of or possibly behind the uterus will be felt a swelling of soft consistence, movable and tender to the touch. The ovary may or may not be felt as distinct from this swelling. 
In cases that have ruptured, intraperitoneally or extraperitoneally, or have aborted, completely or incompletely, examination will seldom carry the diagnosis to a certainty of what has taken place. The bimanual examination will reveal a soft, diffuse, or circumscribed swelling in the pelvis, separate from the uterus and displacing that organ to one side, backwards or forwards, according to the position of the swelling. The swelling usually has a very characteristic feel of a "boggy" nature, and pressure on it causes considerable pain.

An intra-peritoneal rupture usually produces symptoms more severe than an extra-peritoneal one, although it must be borne in mind that the symptoms produced by an extra-peritoneal rupture may be very severe, whereas those produced by an intra-peritoneal rupture may be slight.

$\Delta \mathrm{n}$ intra-peritoneal rupture tends to collect more behind the uterus than to the side, as is the case with an extra-peritoneal rupture. This fact, taken in conjunction with the attendant uterine displacement, will allow of a provisional diagnosis being made.

In forming the diagnosis it is of course correct to say a ruptured tubal pregnancy, whether the rupture has taken place through the tube wall, intra- or extra-peritoneally, or whether it has taken place into the tube lumen, producing an abortion complete or incomplete, as in any case there is a rupture, in the one case through the tube wall and in the other through the limiting membrane into the tube Iumen. The diagnosis has therefore to be between an unruptured and a ruptured tubal gestation.

III. The most LIKely things that May BE CONFUSED WITH aF Extra-diterine Pregnancy.

\section{(1) Intra-uterine Pregnancy.}

It may be extremely difficult to diagnose an intra-uterine pregnancy, especially if accompanied by some small tumour in the pelvis, from an extra-uterine gestation. Every point in the case has to be taken into account and carefully weighed, and even with this it sometimes takes a considerable time of watching the patient and periodically examining her to make quite certain as to the nature of the case.

In one case of my series, a patient with all the symptoms of an extra-uterine gestation, and on examination the feeling of a soft swelling not apparently incorporated with the uterus, was admitted for operation. The symptoms not being urgent, the patient was kept under observation, with the result that after six weeks careful watching, it was determined that the pregnancy was intra-uterine, 


\section{Milligan: Extra-Uterine Pregnancy}

although there was something anomalous about the condition. Later on she was delivered normally of a healthy female child. This case recalls a remark in Bar's ${ }^{1}$ paper, on what he calls pseudo-cornual pregnancy, to the effect that, in many cases, where part of the organ becomes relatively soft, it gives the impression on palpation as though it were movable and therefore a tumour separate from the uterus. Such a condition has led to the diagnosis of extra-uterine gestation more than once. The origin, says Bar, is in the insertion of the ovum close to the ostium of the tube in a normal uterus. Mortagne ${ }^{9}$ reports a case in which a woman suffered from violent abdominal pains, with losses of blood and the discharge of pieces of decidua, and on examination two distinct tumours were thought to be recognizable, and yet the case proved to be one of normal pregnancy. These facts, taken along with the remembrance that an intra-uterine pregnancy may always be complicated by the presence of some tumour in the pelvis, should generally be sufficient to warrant a diagnosis. Should the symptoms point very strongly to the pregnancy as being extrauterine, but yet a certain diagnosis be not possible, there is no question that the passage of a sound is justifiable. This will give valuable information as to whether there is anything in the uterus or not. If it is determined that the uterus is empty, a scraping of the mucosa may be taken and this be examined. A positive result of the presence of decidual tissue clinches the diagnosis of extra-uterine gestation, but it must be remembered that a negative result does not preclude it.

The possibility also has always to be borne in mind that a simultaneous intra-uterine and extra-uterine gestation may be present. The accurrence of this combination greatly masks the symptoms common to each, chief amongst which will be the absence of blood discharge per vaginam, accounted for by the presence of an intrauterine gestation.

\section{(2) Retroversion of the gravid uterus.}

There may sometimes be very great difficulty in diagnosing this from an extra-uterine gestation. A careful physical examination taken in conjunction with the history ought to clear this up. It is in the early months mostly that difficulty in diagnosis arises. The chief symptoms to be depended upon are the urinary symptoms and the bimanual examination. In retroversion of the pregnant uterus, retention is a very common symptom, whereas in extra-uterine gestation it is rare, but pain on passing water is frequent. 
Bimanual examination reveals, in the case of a retroverted gravid uterus, the cervix pointing upwards against the pubes and a soft bulky mass in Douglas's pouch. In extra-uterine gestation where the gestation escapes into Douglas's pouch and there develops, there is the soft mass behind, a cervix pointing downwards and forwards, and unless adhesions are great the body of the uterus ought to be distinguishable from the mass behind. In a case I saw, the history was typical of an extra-uterine gestation, and the physical signs were taken as being corroborative, but laparotomy proved that the case was one of retroversion of the gravid uterus. In this case, had more attention been paid to the hæmorrhagic discharge which was not typical of extra-uterine gestation, and to the fact that the cervix was softer than it would have been likely to be in an extra-uterine gestation of corresponding age, I think the diagnosis would have been more correctly made.

\section{(3) Inflammatory and other acute abdominal conditions.}

(a) Pyosalpinx often presents symptoms very much like those of an extra-uterine gestation. In these cases of pyosalpinx the temperature chart, the pulse, and the pain generally afford pretty definite evidence. Menstrual symptoms may be very misleading, and reliance must be placed on the absence of the signs of pregnancy, and the presence of rigors, temperature, and signs of inflammatory trouble. In certain cases of pyosalpinx the latter symptoms are not present, and then the diagnosis may become very difficult. In such cases the previous history is of importance, as in the case of a pyosalpinx there will be a longer period of continual suffering than is likely to be the case in an early extra-uterine pregnancy.

A point with regard to the temperature must always be remembered, and that is that in cases of hæmatocele a rise of temperature is usually found. It is possible for such a rise to reach several degrees and therefore closely to simulate the temperature of a pyosalpinx.

The following case illustrates very clearly the difficulties that may be met with. A patient with two months' amenorrhoa was attacked with sudden sharp pain in the abdomen, which recurred every now and again, and was accompanied by a discharge of dark thick blood. The temperature was raised and the pulse quickened. Examination revealed the breasts in a state of activity and a large tender swelling to the left of the uterus. A diagnosis of extra-uterine gestation was made, but laparotomy proved the case to be one of pyosalpinx.

(b) Cellulitis. This again may form a difficulty in diagnosis, 
more especially if there has recently been an incomplete abortion.

In favour of the inflammatory condition as being the correct diagnosis, there are the temperature changes, pulse rate, and tenderness on examination, and in those cases where there has been no abortion, the absence of the signs of pregnancy.

(c) Appendicitis. This may present symptoms which are extremely difficult to diagnose from those presented by an extrauterine gestation. There is pain; the onset is sudden; a lump is felt abdominally, which on pelvic examination proves to be by the side of the uterus. The menstrual history may be irregular and afford no real guide as to the condition; the temperature chart and pulse may be deceptive. In such cases all that can be done is to give due prominence to all the important symptoms and to regard syncopal attacks as pointing to internal hæmorrhage, and any marked resistance over the right iliac region as pointing to appendicitis.

Legueu ${ }^{8}$ publishes a very instructive paper on this subject, in which he points out the importance of the presence of signs of syncope, acompanied with no marked contracture of the muscles of the right groin, as being strongly in favour of a diagnosis of extrauterine pregnancy.

(d) Rupture of any abdominal viscus into the peritoneal eavity may simulate very closely the rupture of a gravid tube. Here the history of the case taken along with the symptoms found on examination and the absence of signs of pregnancy, will usually afford a provisional diagnosis, which the necessary laparatomy will clear up.

(e) Intestinal obstruction. There are cases on record where a laparotomy has been performed to relieve an intestinal obstruction, but which have turned out to be cases of extra-uterine gestation. (Cf. Gilford. ${ }^{6}$ )

In such cases a diagnosis will usually be possible, but that there are cases of extra-uterine pregnancy in which it seems well nigh impossible to suspect their true character, is a fact which has to be borne in mind.

Tumours.

(a) Ovarian. Small ovarian tumours with twisted pedicles present symptoms almost identical with extra-uterine gestation. The following case illustrates this fact. A patient, fifteen days over her proper period was attacked with severe pain in her right side, and a blood discharge per vaginam commenced. The discharge lasted four days but the pain continued. On examination a swelling was felt to the right of the uterus, and she was considered to be suffering 
from an extra-uterine gestation. Laparotomy however proved the case to be one of ovarian tumour with a twisted pedicle.

In the above case, had more attention been given to the fact that the blood discharge had lasted only four days, the time of an ordinary period, the diagnosis might have been different.

Ovarian tumours accompanied by intra-uterine pregnancy may present considerable difficulty in diagnosis. Usually the amenorrhna, and the fact that the enlargement of the uterus is too great for an extra-uterine gestation enlargement, are sufficient to guide one aright in diagnosing this condition. The rupture of an ovarian tumour, just as the twisting of its pedicle, may set up symptoms almost identical to those of an extra-uterine gestation.

Then again, ovarian tumours occurring along with an incomplete intra-uterine abortion, may simulate very closely an extra-uterine pregnancy. In all these cases the history taken along with the physical examination will generally lead to a correct diagnosis.

(b) Fibromyoma and fubrocystic tumours. These as a rule present no difficulty, as in the case of a fibromyoma there is usually a long history, owing to its slow growth, and examination reveals a hard swelling, much denser than in the case of a extra-uterine gestation.

In the case of fibrocystic tumours, the difficulty of diagnosis comes in more especially if the tumour is intra-mural, and then the resemblance to an interstitial pregnancy may be great. The absence of signs of pregnancy will usually be sufficient for a correct diagnosis to be made.

Taylor records a case where myoma was diagnosed, but which turned out to be a peritubal hæmatocele around an early tubal pregnancy. The history here, says Taylor, of an acute abdominal pain, very unusual in myoma, should have been sufficient to rouse suspicion of something other than myoma.

(c) Hamatocele. Intra-peritoneal hæmatocele may be caused by other things besides an extra-uterine pregnancy. In ccrtain diseases of the ovaries, there may be severe bleeding into the peritoneal cavity, but it is impossible to formulate any rules as to guidance in the diagnosis, as the symptoms of such disease are not known.

Then there is the condition called metrorrhagic hæmatocele, described by Bernutz and Goupil ${ }^{2}$ as being due to bleeding into the peritoneum from the uterus along the tube. Again, certain blood diseases may cause the tubes to bleed into the peritoneum; but in all these conditions there are usually symptoms of the primary condition present, which taken along with the absence of any signs of 
pregnancy, will usually lead to a correct diagnosis. In any case all such cases must be most carefully gone into and every endeavour made to arrive at a correct diagnosis, as treatment will be largely guided by what is considered to be the condition present.

(d) Extra-peritoneal homatocele or homatoma. There is a difference of opinion as to the frequency with which this is produced by an interrupted tubal gestation. J. W. Taylor and Cullingworth regard it as a rare cause, whereas Doran and Herman regard it as a not infrequent cause. It may follow operations on the broad ligament, and is said to result from rupture of varicose veins. In any case, in the matter of diagnosis, the condition has to be kept in mind and all the symptoms carefully weighed for or against an extra-uterine gestation.

(e) Homatosalpinx. That hæmatosalpinx may result from other causes than an extra-uterine gestation is certain, although it is by no means worked out in what way these hæmatosalpinges occur. There are cases where the possibility of a pregnancy can be excluded with almost a certainty, and in these the occurrence of symptoms pointing to a hæmatosalpinx will receive due consideration, otherwise so far as our present knowledge goes it is impossible to do more than diagnose a hæmatosalpinx and believe that it is due to an extra-uterine gestation.

Such then are the most likely conditions that may be confused with an early extra-uterine gestation. It is essential that every point in the history and physical examination of a doubtful case be taken into account and thoroughly investigated. Pregnancy occurring in a malformed uterus has not been touched upon as such is more likely to be confused with an interstitial pregnancy, a subject on which this paper does not touch.

\section{REFERENCES.}

1. Bar. Bulletin de la Soc. d'Obstét. de Paris, March 20, 1902.

2. Bernutz and Goupil. N.S.S. Transl., vol. i., p. 207.

3. Cullingworth. (1) "Tubal Gestation : an address before Oxford Medical Society, Nov. 12, 1897. (2) Trans. Obstet. Soc., vol. xlvii., p. 411.

4. Dakin. Trans. Obs. Soc., Thond., vol. xxxviii., p. 385.

5. Eden. Trans. Obs. Soc., Lond, vol. xxxix., p. 132.

6. Gilford. Lancet, June 24, 1899, p. 1709.

7. Griffith. Trans. Obs. Soc., Tond., vol. xxxvi., p. 335.

8. Legueu. Bulletins et Mém. de la Soc. de Chir. de Paris, 1902, No. 31.

9. Mortagne. Rev. de Gynécol., vol. viii., p. 361.

10. Taylor. "Extra-uterine Pregnancy," 1899. 